\title{
Availability Assessment of GPS Augmentation System Using Geostationary Satellite and QZSS in Seoul Urban Area
}

\author{
By Kyungho Yoo, ${ }^{1)}$ Sangkyung Sung, ${ }^{1)}$ Eunsung LeE, ${ }^{2)}$ Sanguk LeE, ${ }^{3)}$ Jaehoon KIM, ${ }^{3)}$ \\ Ho-Jin LEE $^{3)}$ and Young Jae LEE ${ }^{1)}$ \\ ${ }^{1)}$ Department of Aerospace Information Engineering, The Konkuk University, Seoul, Korea \\ ${ }^{2)}$ Korea Aerospace Research Institute (KARI), Daejeon, Korea \\ ${ }^{3)}$ Electronics and Telecommunications Research Institute (ETRI), Daejeon, Korea
}

(Received September 17th, 2008)

\begin{abstract}
Today Global Navigation Satellite Systems (GNSS) are widely used for determining position. Within the city environment, however, in which there are many tall buildings, GNSS signals are frequently interrupted, making it difficult for users to obtain information on their exact position. This paper analyzed the availability and positioning performance of a GPS augmented system within the Seoul urban area using the Japanese QZSS (Quasi-Zenith Satellite System) and a geostationary satellite. A 3-dimensional reproduction of Seoul that was based on the 3D GIS (Geographic Information System) Digital Map and a satellite tracking algorithm using Ray-Triangle Intersection algorithm were discussed. A satellite tracking algorithm was verified through filed testing and the QZSS orbit simulator was realized using Keplerian parameter. DOP (Dilution of Precision) and availability in the urban area of Seoul were analyzed using a GPS/QZSS/geostationary satellite separately and simultaneously.
\end{abstract}

Key Words: GPS, QZSS, Geostationary Satellite, 3D GIS Map

\author{
Nomenclature \\ $V:$ direction vector \\ D: distance \\ $a$ : semi-major axis \\ $e$ : eccentricity \\ $v$ : true anomaly \\ $M$ : mean anomaly \\ $u$ : eccentric anomaly \\ $G$ : universal gravitational constant \\ Subscripts \\ $r$ : receiver \\ $d$ : intersecting point \\ $x: x$-axis \\ $y: y$-axis \\ $z$ : $z$-axis
}

\section{Introduction}

The GPS (Global Positioning System), first developed by the US DoD, has been implemented globally to provide position information to users. After its introduction, Russia developed the GLONASS system but is currently operating only 18 satellites for economic reasons, while the EU's Galileo and China's COMPASS are currently in development.

The accuracy of the GPS is strongly influenced by conditions near the receiver. For example, satellite signals are limited in urban areas with skyscrapers or in mountainous regions, causing different problems in providing exact position information to users. Recently, there have been devel-

C 2009 The Japan Society for Aeronautical and Space Sciences opments in GPS research aimed at overcoming these limitations. Simultaneous use of GPS and Galileo increases the availability in urban areas, ${ }^{1)}$ and there has been additional research to improve the position accuracy through by using of GPS and pseudolites. ${ }^{2,3)}$ 3D digital mapping based the DOP simulation system has been developed using GPS and QZSS, ${ }^{4)}$ and current research is also focusing on increasing the availability of QZSS using augmented GPS systems within urban city areas. ${ }^{5,6)}$ Suh and Shibasaki evaluated the effectiveness of QZSS on improving positioning accuracy by simulation by using ray-tracing methods and a 3D digital map. ${ }^{7)}$

This paper provides, a 3D model of Seoul using information from the 3D GIS maps, and analyzes the availability and improvement of positioning performance of augmented GPS systems from the point of a DOP using a QZSS and Geostationary Satellite. A Keplerian parameter is implemented to simulate the orbit of QZSS, provided by JAXA, ${ }^{8)}$ and a 3D modeling methodology is described using a 3D GIS digital maps. In addition, the algorithm that enables determination of whether a line of sight is blocked within an urban city environment is found by application of the Ray-Triangle Intersection algorithm, ${ }^{9}$ used widely in 3D physical and game engines. Results were verified by field testing.

\section{3D Model of Seoul City}

\subsection{Basic elements for building 3D model}

Urban areas are composed of buildings, sewer systems, roads, trees and other land features on a complex topography, requiring some effort and time to visualize the scene. 
Recently, the government of Seoul City has been collecting geographical information to construct a 3D city model. However it has yet to integrate all the information into one single database. Therefore, under current conditions, latitude information of the city is added to a $2 \mathrm{D}$ map to create a $3 \mathrm{D}$ city model. This research assumes the following in creation of a simplified 3D city model.

- There are no landmarks other than buildings in the city.

- The longitude of the buildings is not considered.

- All buildings are box-shaped.

- All buildings are composed of triangular polygons.

Based on these premises, a near-actual 3D city model can be created.

\subsection{Digital map and geodetic system}

To describe the location of an object in 3D space, a 3D plane is needed. The most convenient plane representing the earth is called a geoid. However the geoid is affected by the Earth's gravity. A shape for the Earth that can be expressed mathematically is also needed, reference as an ellipsoid. This ellipsoid is similar in shape to the Earth because it is not an identical replica, countries have developed an ellipsoid size and shape to best express their own location on the Earth.

When applying these local reference systems, a great amount of deviation can be found from one country to another. Hence a unified WGS (World Geodetic System) became essential for use in geodesy and satellite based navigation. The reference system used by the GPS is called WGS84. It is geocentric and is globally consistent within $\pm 1 \mathrm{~m}$. In Korea and Japan, the Bessel 1841 ellipsoid was most commonly used, believing that if best expressed the regional conditions. The characteristics of the Bessel 1841 and WGS84 ellipsoids are summarized in Table $1 .{ }^{10)}$

To indicate $3 \mathrm{D}$ position on a flat map, the coordinates must first be transformed using the transformation parameters between the WGS84 and bessel ellipsoid. Then, the transformed coordinates are projected on the flat map using a TM (Transverse Mercator) projection. This research used coordinate transformation parameters from the National Geographic Information Institute for the coordinate transformation. ${ }^{11)}$

\subsection{Result of 3D modeling of urban canyon}

To produce a 3D model of Seoul, the heights of the buildings were combined in 2D maps. The central point of the coordinate system in the GIS digital map is the central origin $\left(\mathrm{N} 38^{\circ}, \mathrm{E} 127^{\circ}\right)$. A data format was generated as shown in Table 2. The value of the central origin of the $X$-axis is $500,000 \mathrm{~m}(\mathrm{~N})$ and of the $Y$-axis is $200,000 \mathrm{~m}(\mathrm{E})$.

Using the data format in Table 2, the process of generating the 3D building is shown in Fig. 1. The ID value of the building was used to differentiate buildings. Using the value of the $X$ and the $Y$ axis of the building, the surface shape was created and then the height information was combined to construct the final 3D shape. A 3D visual of the entire city was obtained by repeating this process. Figure 2 shows the $3 \mathrm{D}$ model of the $1 \times 1 \mathrm{~km}$ region near Kangnam Station found by repeating the process described above.
Table 1. Detailed field test.

\begin{tabular}{llc}
\hline Place & \multicolumn{2}{l}{$\begin{array}{l}\text { Chamdong Elementary School Sports Ground } \\
\text { (Seoul, Chamshil) }\end{array}$} \\
\hline Date & 10. 08. 2007 & 12:00-17:00 \\
\hline \multirow{3}{*}{ Location } & Lon. (deg) & N 37.517914 \\
& Lat. (deg) & E 127.1003594 \\
& Height (m) & 19.8 \\
\hline
\end{tabular}

Table 2. Orbital parameters of QZSS. ${ }^{10)}$

\begin{tabular}{ll} 
Semi-major axis (m) & $42,164,136$ \\
Eccentricity & 0.099 \\
Inclination (deg) & 45 \\
RAAN (deg) & $0,120,240$ \\
Argument of Perigee (deg) & 270 \\
Mean Anomaly (deg) & $119.76,239.76,359.76$ \\
\hline
\end{tabular}

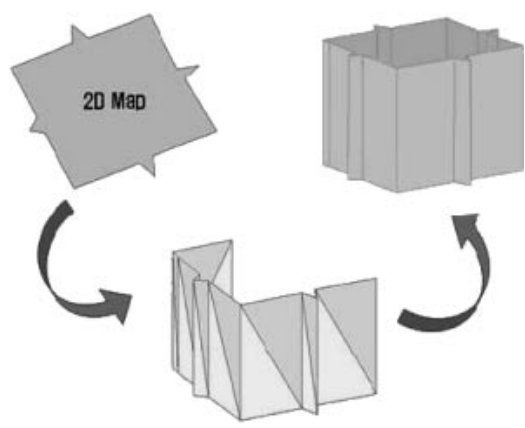

Fig. 1. 3D modeling using 3D GIS map.

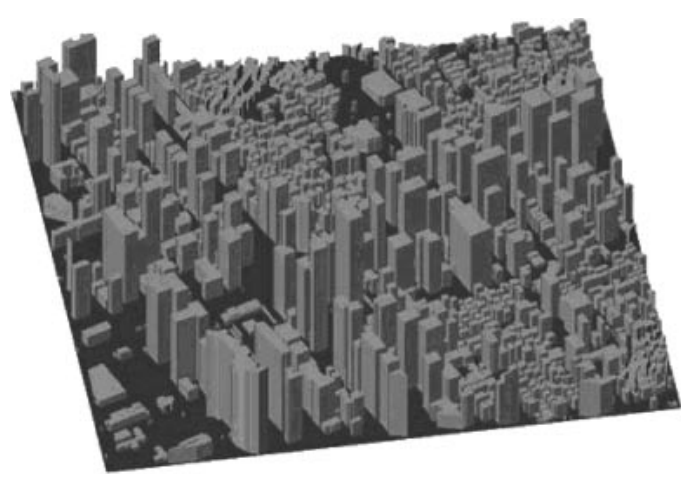

Fig. 2. Result of 3D modeling near Kangnam Station, Seoul

\section{Analysis of Visibility in Urban Canyon}

\subsection{Satellite observation algorithm}

To determine visible satellites in the city, the line of sight blocked by the buildings must be considered. This is similar to the problem of determining the intersection between the defined-line and the plane in a 3D space. This can be achieved using the Ray-Triangle Intersection algorithm, widely used in 3D physical and game engines, virtual reality simulators and other fields. ${ }^{9}$ 


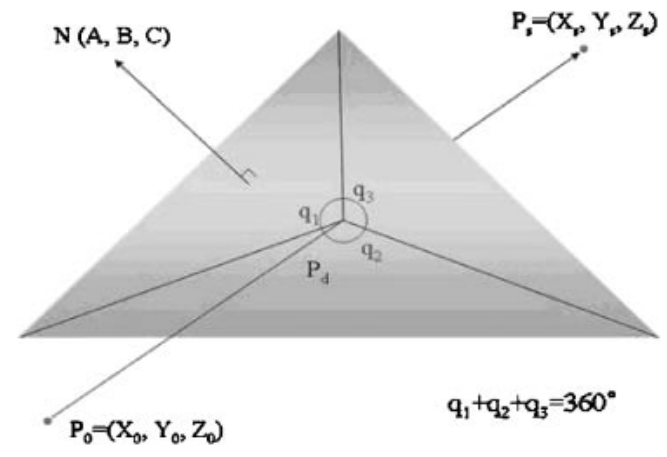

Fig. 3. Ray-Triangle intersection algorithm.

Figure 3 shows typical Ray-Triangle Intersection algorithm. When there are arbitrary two points and a plane with three vertexes within a 3D space, it can be used to determine whether the line connecting these two points intersects the plane. Here, the two points represent the receiver and satellite, and the plane represents the building surface. The intersection test is comprised of a total of three stages, with each stage determining whether there has been an intersection or not. When the results of the tests meet the conditions, it moves to the next stage, and the straight line and plane have intersected when it meets the results of the final stage.

Inspection of possible intersection between line and plane

In the first stage, this determines whether the two points under consideration are positioned on both sides of the plane. The equation of the plane in the 3D space is:

$$
A x+B y+C z+D=0
$$

The vertical unit vector of the plane is defined as $\mathrm{N}$, and the direction vectors in the $x$-, $y$-, and $z$-axis are $A, B$, and $C$, respectively. $D$ can be calculated by applying an arbitrary point on the polygon to Eq. (1). When applying the two points defining the straight line to the equation and retrieving the opposite signs, the two points are found to be on opposite directions of the plane.

\section{Calculation of intersecting point on plane}

After successfully passing the first step, there is a high probability that an intersecting point might exist on the plane. The equation for the straight line can be calculated as:

$$
P_{d}=P_{r}+d \times V
$$

Where, $P_{d}$ represents the intersecting point of the plane, $P_{r}$ is the position of the receiver, and $d$ and $V$ represent the distance to the intersecting point form $P_{0}$ and the direction vector, respectively. Equation (2) can be expressed as:

$$
\begin{aligned}
& P_{d_{x}}=x_{r}+d \times V_{x} \\
& P_{d_{y}}=y_{r}+d \times V_{y} \\
& P_{d_{z}}=z_{r}+d \times V_{z}
\end{aligned}
$$

If there is an intersecting point between the straight line and the plane, Eq. (3) become the $x, y$, and $z$ position of the intersection point, respectively. When applying this to Eq. (1), the equation of the plane can be calculated as:

$$
D=-\left(A \cdot P_{d_{x}}+B \cdot P_{d_{y}}+C \cdot P_{d_{z}}\right)
$$

Equation (6) can be rewritten as:

$$
d=\frac{-\left(A x_{r}+B y_{r}+C z_{r}+d\right)}{\left(A V_{x}+B V_{y}+C V_{z}\right)}
$$

When the straight line and plane are parallel, the denominator becomes 0 . Therefore, when calculating the distance between the receiver and the intersection point, if the denominator is not 0 , the intersection between the straight line and plane can also be calculated by using Eq. (2).

\section{Inspection of intersection point}

This is the step for checking the existence of the intersection point on the plane. First, obtain the vectors from the intersection point to each vertex and then calculate the angle between vectors. If these angles add up to $360^{\circ}$, the point is deemed to be on the plane. If the vectors from the intersection point to the vertexes are named $V_{1}$ and $V_{2}$, the inner product of the two vectors is:

$$
V_{1} \cdot V_{2}=\left|V_{1}\right|\left|V_{2}\right| \cos q
$$

In Equation (8), $q$ represents the angle calculated by vector $V_{1}$ and $V_{2}$. Equation (8) can be expressed as follows.

By using Equation (9), we can obtain the angle between two vectors. If the sum of the angles is $360^{\circ}$, the intersection exists on the plane.

$$
q=\cos ^{-1}\left(\frac{V_{1} \cdot V_{2}}{\left|V_{1}\right|\left|V_{2}\right|}\right)
$$

The positions of the simulation points can be determined from a 3D digital map and the positions of the satellites are calculated using the ephemeris data. Therefore, if a 3D model of the city can be created, it is possible to determine the visibility in the urban area using this algorithm.

\subsection{Verification of algorithm using field testing}

A field test was conducted to verify the satellite tracking performance using the Ray-Triangle Intersection algorithm. The algorithm was verified by comparing the number of visible satellites calculated from the simulation with observed satellites in the field. An algorithm was verified. The area where trees and other geographical non-building figures had little or no affect on the satellite signal was determined as the area for the test. In addition, to guarantee the most accurate results, buildings that were not indicated on the digital map were added through actual on-sight observation and verification. The results of the test are summarized in Table 1.

The test was conducted as a static test for five hours between 12:00 to 17:00 on 8 August 2007. GPS data was received at three minute intervals. Figure 4 shows both the results of the field test and the simulation. The field test and simulation showed a very similar tendency between visible satellites. When creating the $3 \mathrm{D}$ model, there were some discrepancies through the presumed information, but it had no significant effect. 


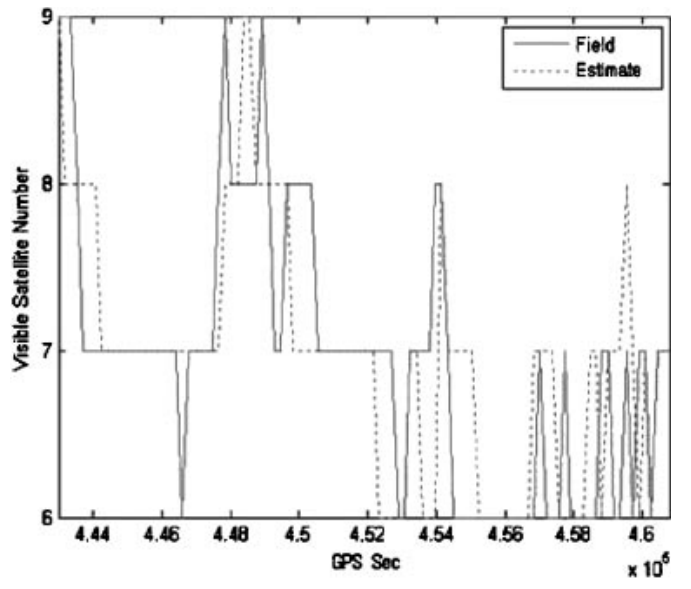

Fig. 4. Comparison of the results of the field test and the simulation.

\section{Quasi-Zenith Satellite System}

\subsection{QZSS introduction}

The Quasi-Zenith Satellite System (QZSS) project began as a program to supplement the limitations of GPS accuracy through the increased need for GPS-related technologies including automobile navigation and GPS installed on cell phones in Japan. QZSS is composed of a minimum of three different satellites with different orbits, and just like geostationary satellites, rotate about the Earth in an orbit of 23 hours 56 seconds. The elevation of GPS geostationary satellite is about $45^{\circ}$ but the QZSS satellite has an elevation greater than $70^{\circ}$. Although the signals from GPS geostationary satellite can be blocked by city buildings, QZSS with a relatively greater elevation can be detected even within downtown. Therefore, when this is implemented with GPS, the number of detectable satellites will increase availability and position accuracy. There is a good possibility of enhancing the position accuracy for users in Korea, which is also within the QZSS domain using this method.

\subsection{QZSS orbit simulation}

To calculate the coordinates for the QZSS satellite, the orbit was simulated using the Keplerian equation representing the relative motion of two objects. The Earth and QZSS satellite can be expressed as:

$$
\ddot{r}=\frac{-G\left(m_{1}+m_{2}\right)}{r^{3}} \vec{r}=-\mu \frac{\vec{r}}{r^{3}}
$$

Equation (10) is the equation of Newtons' vector motion, representing the relative motion of large and small objects. This equation can be applied to determine the orbital equation.

$$
\vec{r}=\frac{a\left(1-e^{2}\right)}{1+e \cos v}
$$

Equation (11) is derived from the orbital equation in Equation (10), where a represents the semimajor-axis, $e$ represents eccentricity, and $v$ represents true anomaly. The results and the characteristics of the ellipsoid orbit are shown in Fig. 5. When Eq. (11) is solved for time, the following equation is obtained:

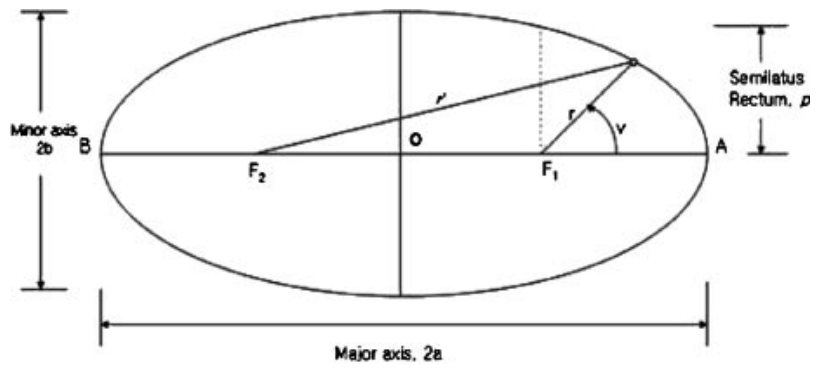

Fig. 5. Major characteristics of ellipse.

$$
t=\sqrt{\frac{a^{3}}{\mu}}(u-e \sin u)
$$

Where, $u$ represents eccentric anomaly, if mean anomaly is expressed as M, we can this in the following Keplerian equation.

$$
M=u-e \sin u
$$

The orbital parameter determining the QZSS orbit is summarized in Table $4 .{ }^{10)}$ Using the QZSS orbital parameter, the ground trajectory and variation in elevation were simulated; the results are shown in Fig. 6 and Fig. 7 respectively. As shown in Fig. 7, three QZSS in Seoul has an elevation greater than $70^{\circ}$ at all times at a specific position (N 37.6296936944 $\left.{ }^{\circ}, \mathrm{E} 127.0797240833^{\circ}, 59.109 \mathrm{~m}\right)$.

\section{Simulation Results}

Based on the 3D model of the region near Kangnam Station, Seoul, (an area with a concentration of high buildings) the position information from over 3000 roads and side streets was used to analyze availability and DOP function. The simulation was conducted for 8 different scenarios and combined the QZSS with geostationary satellites for 24 GPSs. The positions of the GPS satellites were based on data provided by the IGS (International GNSS Service), and the position of the QZSS satellites were calculated using the orbit simulator. The positions of the geostationary satellites were calculated using three orbit slots $\left(\mathrm{E} 113^{\circ}\right.$, $\left.\mathrm{E} 116^{\circ}, \mathrm{E} 126^{\circ}\right)$ from the four orbit slots $\left(\mathrm{E} 113^{\circ}, \mathrm{E} 116^{\circ}\right.$, $\left.\mathrm{E} 126^{\circ}, \mathrm{E} 129^{\circ}\right)$ designated to Korea. The elevation mask was set at $10^{\circ}$, and the simulation was conducted for an entire day at 15-minute intervals (29-30 December 2007 00:00-00:00 UT).

\subsection{Satellite availability}

The results for the availability obtained from the independent and combined application for the GPS/QZSS/ Geostationary satellite in Seoul are shown in Fig. 8.

Table 3 summarizes the results for the availability simulation. The position was set with over four observed satellites as the available area. Availability in the city center using the GPS/QZSS/Geostationary satellites increased. In the case of GPS usage alone, areas with $0-80 \%$ availability level covered $24.4 \%$ of the entire testing area, but this area was greatly reduced when the geostationary satellite 
Table 3. Results of the available ratio test per day.

\begin{tabular}{lrrrrr}
\hline \multicolumn{1}{c}{ Integrate option } & $0-20 \%$ & $20-40 \%$ & $40-60 \%$ & $60-80 \%$ & $80-100 \%$ \\
\hline GPS & $24.4 \%$ & $13.7 \%$ & $11.4 \%$ & $12.2 \%$ & $38.3 \%$ \\
GPS + GEO1 & $21.4 \%$ & $11.2 \%$ & $8.8 \%$ & $9 \%$ & $49.6 \%$ \\
GPS + GEO2 & $19.6 \%$ & $10.3 \%$ & $7.6 \%$ & $6.5 \%$ & $56 \%$ \\
GPS + GEO3 & $17.2 \%$ & $7.3 \%$ & $6.1 \%$ & $4.9 \%$ & $64.5 \%$ \\
GPS + QZSS & $7.4 \%$ & $3.6 \%$ & $6.8 \%$ & $8.5 \%$ & $73.7 \%$ \\
GPS + GEO1 + QZSS & $6.4 \%$ & $3.4 \%$ & $6.2 \%$ & $7.4 \%$ & $76.6 \%$ \\
GPS + GEO2 + QZSS & $6.1 \%$ & $3.1 \%$ & $5.9 \%$ & $6.8 \%$ & $78.1 \%$ \\
GPS + GEO3 + QZSS & $6 \%$ & $3.1 \%$ & $5.4 \%$ & $15.5 \%$ & $80 \%$ \\
\hline
\end{tabular}

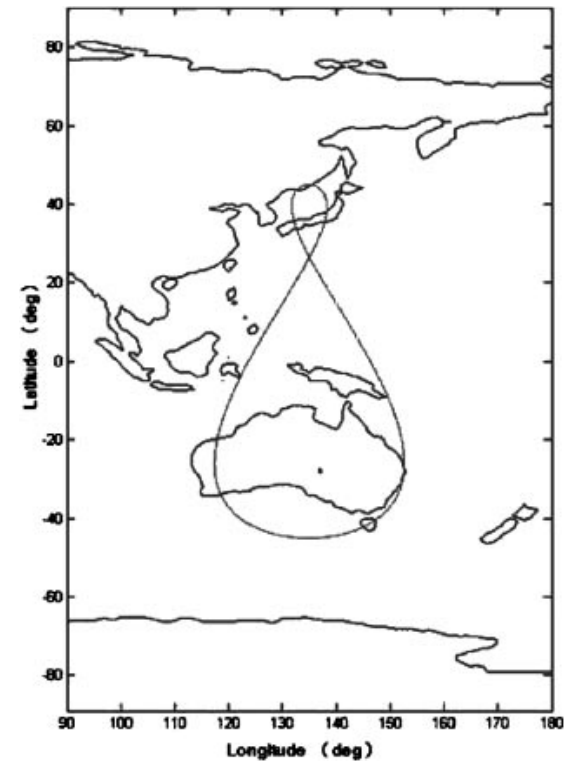

Fig. 6. Ground trajectory of QZSS.

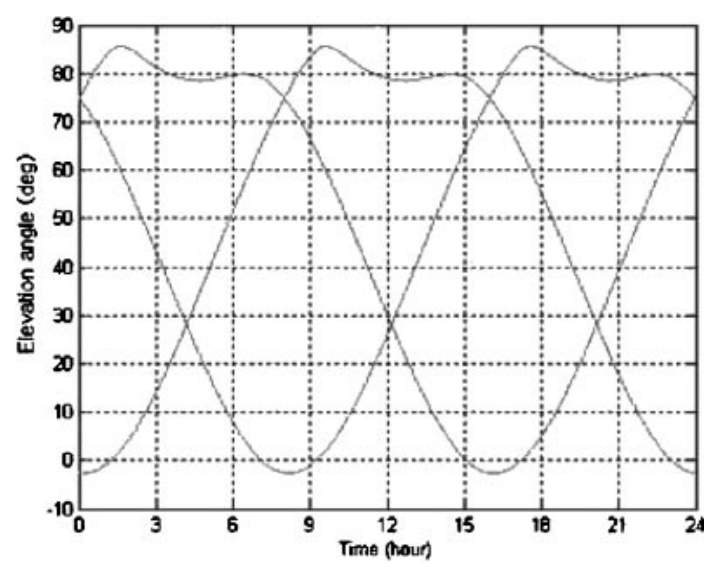

Fig. 7. Variation of the elevation angle for Seoul.

and QZSS were used together. Areas with 80-100\% availability were only detected in $38.3 \%$ of the total area that used GPS only but in $80 \%$ of the area using both geostationary satellite and QZSS, representing a 40\% increment compared to that of the GPS-only region. The availability of GPS augmented with QZSS was better than GPS combined with geostationary satellite, because the QZSS has higher elevation than the geostationary satellite.
Table 4. Results of the HDOP and PDOP test per day.

\begin{tabular}{lcccccc}
\hline \multirow{2}{*}{ Integrate option } & \multicolumn{2}{c}{ HDOP } & & \multicolumn{2}{c}{ PDOP } \\
\cline { 2 - 3 } \cline { 5 - 6 } & Mean & Std dev. & & Mean & Std dev. \\
\hline GPS & 4.93 & 4.30 & & 6.96 & 5.14 \\
GPS + GEO1 & 4.18 & 4.22 & & 6.04 & 4.95 \\
GPS + GEO2 & 4.04 & 4.19 & & 5.86 & 4.89 \\
GPS + GEO3 & 3.60 & 3.95 & & 5.36 & 4.40 \\
GPS + QZSS & 2.77 & 2.03 & & 3.96 & 2.85 \\
GPS + GEO1 + QZSS & 2.57 & 2.02 & & 3.73 & 2.82 \\
GPS + GEO2 + QZSS & 2.52 & 2.02 & & 3.67 & 2.82 \\
GPS + GEO3 + QZSS & 2.42 & 1.99 & & 2.55 & 2.78 \\
\hline
\end{tabular}

\subsection{DOP simulation}

The geometric array of the satellite has a strong affect on the position accuracy for users and this is expressed in DOP (Dilution of Precision). To calculate the DOP, the following equation is used. ${ }^{11)}$

$$
\begin{aligned}
\left(G^{\mathrm{T}} G\right)^{-1} & =\left(\left[\begin{array}{cccc}
l_{1} & l_{2} & \ldots & l_{k} \\
m_{1} & m_{2} & \ldots & m_{k} \\
n_{1} & n_{2} & \ldots & n_{k} \\
1 & 1 & \ldots & 1
\end{array}\right]\left[\begin{array}{cccc}
l_{1} & m_{1} & n_{1} & 1 \\
l_{2} & m_{2} & n_{2} & 1 \\
\vdots & \vdots & \vdots & \vdots \\
l_{k} & m_{k} & n_{k} & 1
\end{array}\right]\right)^{-1} \\
& =\left[\begin{array}{cccc}
\sigma_{x x}^{2} & \sigma_{x y}^{2} & \sigma_{x z}^{2} & \sigma_{x t}^{2} \\
\sigma_{y x}^{2} & \sigma_{y y}^{2} & \sigma_{y z}^{2} & \sigma_{y t}^{2} \\
\sigma_{z x}^{2} & \sigma_{z y}^{2} & \sigma_{z z}^{2} & \sigma_{z t}^{2} \\
\sigma_{t x}^{2} & \sigma_{t y}^{2} & \sigma_{t z}^{2} & \sigma_{t t}^{2}
\end{array}\right]
\end{aligned}
$$

Where $l, m$ and $n$ are the vector with unit length from the center of the satellite cell, and $k$ represents the number of visible satellites. Using the diagonal terms shown in the matrix under Eq. (14), DOP is defined as:

$$
\begin{aligned}
& \text { PDOP }=\sqrt{\sigma_{x x}^{2}+\sigma_{y y}^{2}+\sigma_{z z}^{2}} \\
& \mathrm{HDOP}=\sqrt{\sigma_{x x}^{2}+\sigma_{y y}^{2}}
\end{aligned}
$$

This shows that the smaller the DOP value, the more accurate the position for the user becomes. The results of the HDOP test are shown in Table 4 . Table 4 shows the daily mean value and standard deviation for HDOP and PDOP but DOP values greater than 100 were excluded. 


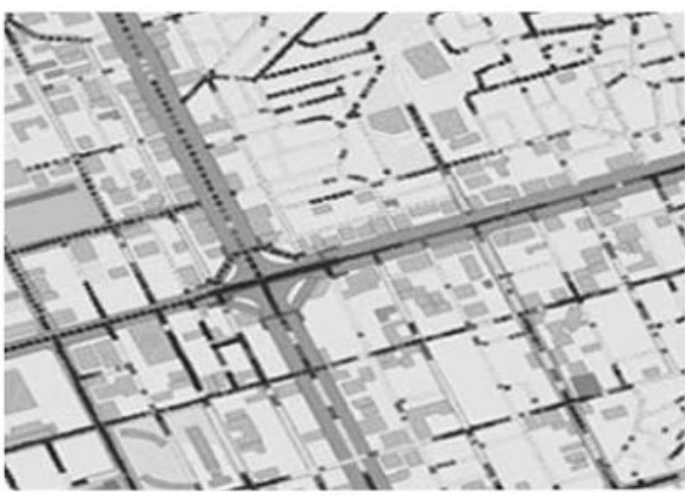

(a)

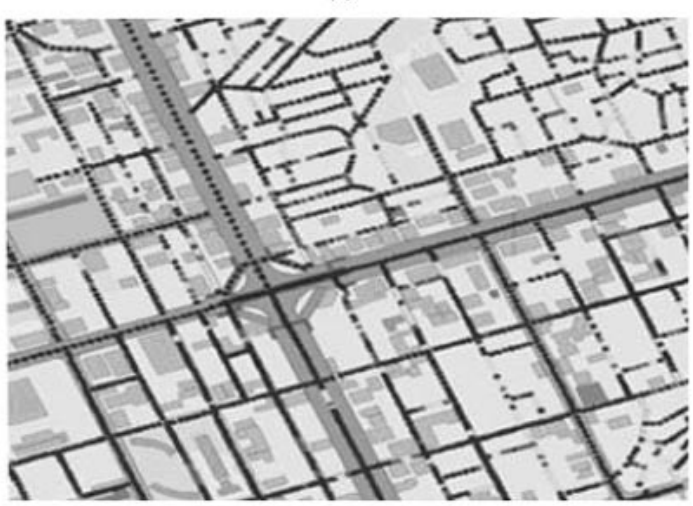

(c)

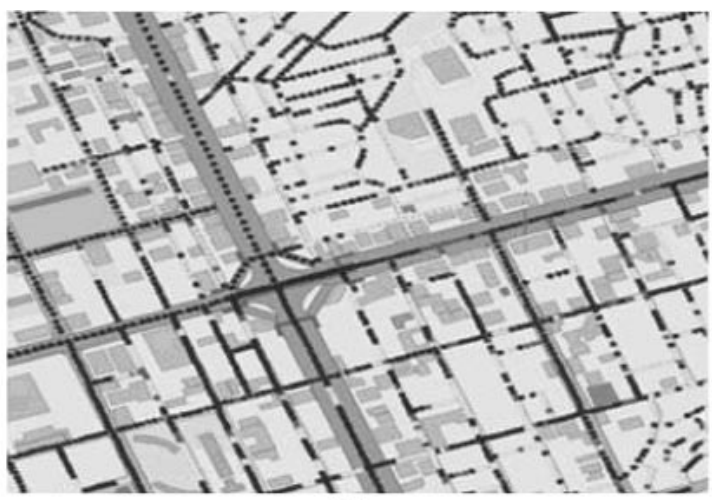

(b)

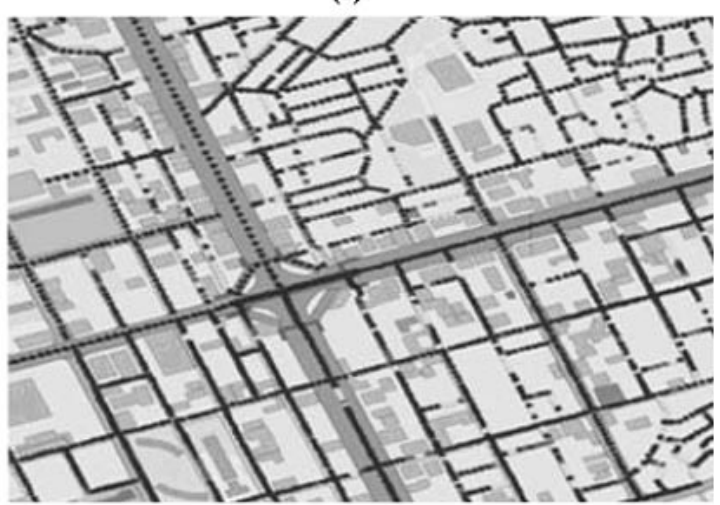

(d)

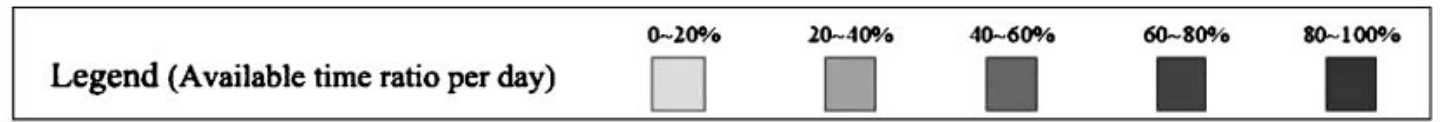

Fig. 8. Availability test through integrated options: (a) GPS Only (b) GPS with 3 geostationary satellites (c) GPS with QZSS and (d) GPS with 3 geostationary satellites and QZSS.

The simulation results show that the combination of GPS and QZSS provides a more accurate position than the results from the combination of GPS and geostationary satellite, because the QZSS has a higher elevation above Seoul, providing a better geometric array. In contrast, geostationary satellites are concentrated within the same longitude $\left(\mathrm{E} 113^{\circ}, \mathrm{E} 116^{\circ}, \mathrm{E} 126^{\circ}\right)$, having a relatively low DOP performance. In addition, as the number of GNSS combined with GPS increases, the HDOP and PDOP decreases, showing both the enhanced availability of the GPS system using the QZSS and geostationary satellite, as well as improved user position accuracy.

\section{Conclusions}

This research analyzed GPS availability and DOP performance in Seoul city center combining a geostationary satellite as well as QZSS with the conventional GPS.

We confirmed that the level of availability increased when GPS is combined with QZSS and geostationary satellites, rather than used alone. The availability of the GPS augmented system increased but the user position accuracy increased too. Finally, using QZSS, with a higher elevation above Seoul than the geostationary satellite, offered a higher level of availability and more accurate user position than when using GPS with a geostationary satellite.

\section{Acknowledgments}

This work was supported by the IT R\&D program of the KCC and the IITA. [2007-S-301-01, Development of GNSS ground station and SAR beacon technologies]. This work was supported in part by the Korea Foundation for International Cooperation of Science \& Technology through a grant provided by the Korean Ministry of Education, Science \& Technology (MEST) in 2008 (No. K20601000001).

\section{References}

1) Jensen, A. B. O., Zabic, M., Overø, H. M. and Nielsen, O. A.: Availability of GNSS for Road Pricing in Copenhagen, ION GNSS 2005, Long Beach, California, USA, 2005, pp. 2951-2961.

2) Stone, J. M. and Powell, J. D.: Precise Positioning with GPS Near Obstructions by Augmentation with Pseudolites, Position Location and Navigation Symposium, IEEE 1998, 20-23 April 1998, pp. 562-569.

3) Konishi, Y. and Shibasaki, R.: Development of a Simulation System to Estimate Available Area of GPS and Pseudolite, 22nd Asian Conference on Remote Sensing, Singapore, 5-9 November 2001.

4) Hakamata, T., Konishi, Y., Suh, Y. and Shibasaki, R.: Development of a Simulation System to Delineate Availability of GNSS with 3-D Digital Map, Asian Conference on Remote Sensing, Session. GIS GPS \& Data Integration, 2002.

5) Suh, Y., Konish, Y., Hakamata, T. and Shibasaki, R.: The Effect of Quasi-Zenith Satellite System in Urban Environments, Geoinformation Student Forum Japan 2003.

6) Suh, Y. and Shibasaki, R.: A Simulation Based Assessment for Eval- 
uating the Effectiveness of Quasi-Zenith Satellite System, Korean J. Remote Sensing, 19 (2003), pp. 181-190.

7) Suh, Y. and Shibasaki, R.: Evaluation of Satellite-Based Navigation Services in Complex Urban Environments Using a ThreeDimensional GIS, IEICE Trans. Commun., E90-B (2007), pp. 18161825

8) JAXA: Quasi-Zenith Satellite System Navigation Service: Interface
Specification for QZSS, retrieved from: http://qzss.jaxa.jp, 2007.

9) Kenine-Moller, T. A. and Haines, E.: Real-Time Rendering, 2nd ed., AK Peters, USA, 2002.

10) Kishimoto, M.: Quasi-Zenith Satellite System: Status and Design, Location, January-February 2007.

11) Hofmann-Wellenhof, B. and Lichtenegger, H.: GPS:Theory and Practice, 5th ed., Springer, Wien, New York, 2000. 